\title{
Erratum to: The stable free rank of symmetry of products of spheres
}

\section{Bernhard Hanke}

Published online: 30 July 2010

(C) Springer-Verlag 2010

\section{Erratum to: Invent math (2009) 178: 265-298}

DOI 10.1007/s00222-009-0197-3

Due to a printing error, which occurred after the galley proofs had been corrected, a part of a sentence in the last but one paragraph on p. 266 in [1] is missing. The complete paragraph should read:

Theorem 1.4 is implied by a theorem of Halperin [17] which yields upper bounds of free toral ranks in terms of homotopy Euler characteristics. The proof is based on rational homotopy theory applied to the Borel space $X_{\left(S^{1}\right)^{r}}$. Hence rational homotopy theory has become an important tool in the study of torus actions in general, see [4, Sect. 2] for a survey.

\section{References}

1. Hanke, B.: The stable free rank of symmetry of products of spheres. Invent. math. 178, 265-298 (2009)

The online version of the original article can be found under doi:10.1007/s00222-009-0197-3.

B. Hanke $(\varangle)$ Institut für Mathematik, Universität Augsburg, 86135 Augsburg, Germany e-mail: hanke@math.uni-augsburg.de 